\title{
Context, integration, and retrieval
}

\author{
LINDA BAKER and JOHN L. SANTA \\ Rutgers University, Douglass College, New Brunswick, New Jersey 08903
}

\begin{abstract}
Three experiments examined the effectiveness of external retrieval cues when encoding context varied with respect to the integration of the representation. In all three experiments, it was found that nonencoded cues led to greater improvement if the initial representation was not well integrated. Strong-associate cues led to more improvement when encoding context consisted of weak-associate pairs than when the pairs were embedded in sentences (Experiment 1). The cues were more effective when subjects studied a list of words without instructions than when they were instructed to form images integrating the list members (Experiment 2). The third experiment demonstrated that well integrated material takes longer to access, and a control experiment argued against an encoding interpretation of the data. The results demonstrated both a flexibility of retrieval and a restriction from context, such that the better the representation, the harder it is to retrieve using external retrieval cues.
\end{abstract}

Organization seems to be the key ingredient for a good memory. This conclusion emerges readily from the past decade of research. Beginning with simple lists, it is found that to organize is to remember (Mandler, 1967). When subjects are given lists containing categorical structure, they use it (Bousfield, 1953), and the more structure, the better (Bower, Lesgold, \& Tieman, 1969). Nor are subjects restricted to categorical and logical organization; stories, imagery, and assorted mnemonic devices, such as the method of loci, are all equally serviceable means to create an organized representation. Each of these methods improves memorability by weaving the individual memories into a well integrated context.

But is it always desirable to embed a memory in such a tightly woven context? The answer is clearly "no" if one accepts as evidence the complaints of students after a psychology examination. All too often we hear that students knew the lecture notes by heart, but failed the test because they were unable to produce the required answers. If allowed to reproduce their notes instead, they could do so, even to the extent of knowing on which page each item is located. Perhaps the students' problem is that they "know" the material too well; it is overly tied to a particular context.

The present paper tries to establish in a laboratory setting this detrimental effect of knowing material too well. We want to demonstrate that the quality of the original encoding can restrict subsequent retrieval.

Order of authors was determined alphabetically. Reprint requests should be addressed to John L. Santa, Department of Psychology, Douglass College, Rutgers University, New Brunswick, New Jersey 08903. This research was in part supported by Rutgers Research Council grants to the second author. We wish to express our appreciation to Linda $L$. Lamwers for her assistance throughout this work. Experiments 1 and 2 were first reported at the Eastern Psychological Association meetings in 1975, and Experiment 3 was reported at the 1976 EPA meetings.
In short, the better integrated the representation, the harder it is to break the original context and to retrieve with different cues.

The issue of context constraint on retrieval is related to at least two recent lines of investigation. First, there is a large set of experiments which suggest that changing context from study to test can be detrimental in a recognition paradigm (Hunt \& Ellis, 1974; Light \& Carter-Sobell, 1970; Tulving \& Thomson, 1971). More to the point, a number of investigators have also shown that the magnitude of the context effect depends upon the quality of the initial representation (Baker \& Santa, 1977; Ciccone \& Brelsford, 1975; Jacoby, 1972; Pompi \& Lachman, 1967; Winograd, Karchmer, \& Russell, 1971).

These recognition data are certainly consistent with the intuition that a well integrated representation is not always an asset. But, does the goodness of context influence the usefulness of external retrieval cues? This aspect of the context effect has been partially examined in a number of experiments by Tulving and his colleagues (Thomson \& Tulving, 1970; Tulving \& Osler, 1968; Tulving \& Thomson, 1973). These experiments have led Tulving and his colleagues to postulate a rather strong principle of encoding specificity, according to which a retrieval cue is useful if and only if present during encoding. Santa and Lamwers $(1974,1976)$ have criticized this work on both methodological and theoretical grounds. Empirically, the cued recall experiments that led to the principle of encoding specificity seemed to depend heavily on the subjects' understanding of the task. With certain instructions, external retrieval cues are useless, but with other instructions, the cues are quite useful. Theoretically, the principle is untestable since it is impossible to know precisely what is or is not encoded at time of study.

In spite of these objections, Tulving's work has directed attention to the important issue of contextual 
effects in memory. Clearly, people are constrained by the circumstances of encoding even if they are not totally bound by encoding context. The interesting questions, then, remain as to the factors that influence retrieval. The present experiments focus on the limits to retrieval flexibility by demonstrating that, as the initial representation becomes better integrated, it is harder to access in different ways.

\section{EXPERIMENT 1}

The first experiment tested context effects on retrieval by using a procedure similar to that used by Tulving and his colleagues. In one condition, subjects studied three lists of 24 weak-associate pairs. This condition was contrasted to one in which each word pair was embedded in a sentence. The sentences were expected to provide better integrated representations of the to-be-remembered (TBR) words than the simpler cue-target pairs. It was, therefore, expected that strongassociate cues would be more useful for the simple pairs than for the better integrated sentences.

\section{Method}

Materials. Three lists of 24 weak-associate TBR pairs were compiled. Two-thirds of the pairs on each list were those used by Tulving and Thomson (1973), while the remaining pairs were obtained from the Bilodeau and Howell (1965) norms using the same selection criteria. Strong-associate cues were also available for each TBR word. Seventy-two short descriptive sentences were constructed that had the weak-cue TBR pairs embedded within them. There were no specific rules of construction with respect to sentence length and structure, but the cue word always preceded the target. As an example, for the pair, "cheese-GREEN," the sentence was: "The cheese was covered with GREEN mold."

The materials were typed and reproduced as transparencies. For the word-pair presentation conditions, the cue appeared in lowercase letters to the left of the TBR, which was capitalized. For the sentence presentations, the cue was again in lowercase letters and the TBR was capitalized; both words were underlined. List presentation order was partially counterbalanced, with one list serving as the test list for some subjects, and the first set list for others. The second set list was the same for all subjects.

Design. The experiment consisted of a 2 by 2 betweensubjects factorial design, with two encoding conditions (word pairs and sentences) and two recall cuing conditions (strongassociate cues and no cues). All subjects were given two set lists, in which they were tested for recall in the presence of the original weak cues. The cuing conditions were defined by type of recall on the third critical list.

Seventy-three Douglass College introductory psychology students participated in the experiment for extra credit. The number of subjects in the four experimental conditions ranged from 14 to 22 .

Procedure. Subjects in the word-pair conditions were instructed to learn the capitalized words and to use the words in lowercase letters to help remember the targets. Subjects in the sentence conditions were also instructed to learn the capitalized words, and they were told that the sentence context and second underlined word would help to make the target more memorable.

The lists were presented with an automated overhead projector. Each word pair or sentence was exposed for $5 \mathrm{sec}$ with a .5 -sec interstimulus interval. Following presentation of the first list, all subjects were given a weak-associate cued recall test. The response sheet contained the 24 study cues intermixed with 12 distractors, included as a control for guessing. Subjects were instructed to write down the capitalized words that had been paired with the cues during study and they were told that some of the words had not been presented during study. The second list was then presented, again followed by a weak cued recall test. The first two tests were intended to induce subjects to encode the capitalized words with respect to the original context, leading them to expect a weak cued recall test on the third list as well.

Following presentation of the third list, subjects were either given a strong-associate cued recall test or a free recall test. Subjects in the cue conditions were informed that the cue words had not been seen before, but that some were related to the capitalized words they had just studied. They were told to go through the list of cues, generating associates to each of them; if a capitalized word came to mind, they were to write it in the corresponding space. They were cautioned that some of the cue words (distractors) would not lead to study items. Subjects in the free recall conditions were simply instructed to write down as many of the capitalized words as they could remember. Four minutes were allowed to complete all recall tasks.

\section{Results and Discussion}

Set lists. The mean number of target items recalled on the two set lists in response to the weak-associate cues is shown in Table 1, collapsed across cue condition which is not relevant. Analysis of variance showed a main effect of encoding condition $[F(1,67)=19.63$, $\mathrm{MSe}=26.03]^{1}$ and of test trial $[\mathrm{F}(1,67)=100.57$, $\mathrm{MSe}=4.58]$ and an interaction of Encoding Condition by Test Trial $[F(1,67)=5.03, \mathrm{MSe}=4.58]$. More targets were recalled when the encoding context consisted of isolated weak-cue TBR pairs than when the pairs were embedded in sentences. The superiority of word pairs can probably be attributed to relative differences in study time. Word pairs and sentences were both presented for $5 \mathrm{sec}$, but, since sentences required more processing than did word pairs, they were probably not as well learned. Recall increased from the first to the second test trial, and sentenceencoding subjects showed a greater improvement over tests than did word-pair subjects, perhaps due to changes in encoding strategy.

Test list. The cued recall protocols were scored by considering a target word as correct even if it was recalled in response to a distractor or a strong-associate cue for another target. (The use of the lenient scoring system did not substantially influence results, adding only .42 additional targets to the mean obtained when items were scored as correct only if recalled in response to the specified strong-associate cue for that target.) The

Table 1

Mean Number of Words Recalled on the Set Lists With Weak-Associate Cues

\begin{tabular}{ccc}
\hline Encoding Condition & Set List 1 & Set List 2 \\
\hline Word Pairs & 16.66 & 19.51 \\
Sentences & 11.98 & 16.47 \\
\hline
\end{tabular}


Table 2

Mean Number of Words Recalled in Experiments 1 and 2

\begin{tabular}{crc}
\hline Encoding Condition & Cued Recall & Free Recall \\
\hline & \multicolumn{3}{c}{ Experiment 1 } \\
Word Pairs & 11.65 & 5.55 \\
Sentences & 8.62 & 5.27 \\
& \multicolumn{3}{c}{ Experiment 2} \\
No Imagery & 19.42 & 11.83 \\
Imagery & 17.73 & 17.50 \\
\hline
\end{tabular}

proportion of nontarget responses made to distractors was very low (mean $=.05$ ), indicating that subjects were not simply guessing when they wrote down associates. The top half of Table 2 shows the mean number of targets recalled on the critical list for the two encoding conditions on free and cued recall. An unweighted means analysis of variance revealed a reliable main effect of cue condition $[F(1,66)=27.76, \mathrm{MSe}=13.46]$. Subjects provided with retrieval cues recalled more targets than did subjects recalling without cues. An analysis of simple effects showed that the cues led to significantly better recall, regardless of encoding condition. The effect of encoding condition approached significance $[F(1,66)=3.41, p=.07, \quad M S e=13.46]$, with recall higher when the encoding context consisted of isolated word pairs. Although the Encoding by Cue Condition interaction failed to reach conventional levels of significance $[F(1,66)=2.36, p=.13$, $\mathrm{MSe}=13.46]$, there was a clear trend toward the predicted effect. Strong-associate cues were more effective when the encoding context consisted of simple word pairs than when the words were embedded in sentences. An analysis of simple effects supports this interpretation: The difference between encoding conditions on cued recall was reliable $[F(1,66)=5.82$, MSe $=13.46]$, while there was no difference between encoding conditions on free recall.

The fact that external cues led to significant improvement for both conditions over free recall is, of course, inconsistent with a complete encoding specificity. Of more central interest is the suggestion that the quality of the encoding context influenced the usefulness of retrieval cues. However, the marginal interaction led to the suspicion that the paradigm provided only a weak test of the hypothesis. One problem was that sentences did not seem to provide a better representation than the word pairs. In both weak cued and free recall tasks, the word condition led to performance that was as good or better than that in the sentence condition. This result was probably caused by the sentence subjects having more material to process; consequently, they may not have learned the material sufficiently well to benefit from the richer encoding context. More importantly, it is possible that a richer encoding context will not affect the usefulness of retrieval cues unless it creates a better integration among items. Presumably, a sentence provides a better representation for a single TBR word, but at the same time, it might prevent the subject from forming an organization among the targets that might lead to improved recall.

\section{EXPERIMENT 2}

The second experiment used a different paradigm designed to eliminate the problems of Experiment 1 and to provide an additional test of the context effect on retrieval. All subjects had identical input materials, but context was manipulated via encoding instructions. Some subjects were instructed to form images interrelating groups of words, while others were left to devise their own encoding strategies. Given the large literature on the efficacy of imagery instructions, it was assumed that this manipulation would lead to superior free recall performance and a better in tegrated representation. However, consistent with our original hypothesis, it was expected that subjects who did not receive imagery instructions would benefit more from nonencoded retrieval cues.

\section{Method}

Materials. The stimulus materials consisted of a single list of 28 concrete nouns, selected from the TBR words used in Experiment 1. Concrete words were used in order to make the materials as imageable as possible. The retrieval cues were the same high associates used in the first experiment.

Design. The four experimental conditions differed with respect to encoding instructions (imagery vs no imagery) and type of recall (cued vs noncued). Subjects in the imagery conditions formed progressive images of groups of four nouns interacting, while the remaining subjects were free to learn the list in any way they chose.

Subjects were 53 introductory psychology students who received extra credit for their participation. The number of subjects in each of the four cells ranged from 12 to 15 .

Procedure. Subjects in the imagery condition were told that they would hear a list of concrete nouns and, as a way to help remember the words, they were to form progressive images in which groups of four items interacted. They were given an example of how this should be done, first by imaging the first word in the group, then by adding the second word to the image when it was presented, followed by the third word, and then the fourth. Subjects in the no-imagery group were simply asked to learn the words as best they could for a later (unspecified) memory test.

The list was presented auditorally, recorded on tape in a male voice. There was a 4-sec presentation rate, with an additional 4-sec pause between groups of four items. The imagery subjects were told that the pause was to allow time to complete the interacting image of the preceding four items. The no-imagery subjects were simply told that the intervals between words would be of varying lengths. Following list presentation, subjects were allowed $4 \mathrm{~min}$ to complete either a cued or free recall task. The cued recall test sheet consisted of 24 cues and 12 distractors. Cues were not provided for the last four input items, which were included on the study list for the purpose of reducing possible recency advantages to free recall subjects. Consequently, these items were ignored when scoring the recall protocols. 


\section{Results and Discussion}

The mean number of words recalled by subjects in the two encoding conditions is shown in the bottom half of Table 2 for both cued and free recall. An analysis of variance revealed that both main effects and the interaction were reliable. The imagery-encoding subjects recalled more words than did noninstructed subjects $[\mathrm{F}(1,49)=3.90, \mathrm{MSe}=12.88]$. This effect is due to the large superiority of imagery subjects on free recall, suggesting that these subjects did have a better initial representation. This claim was supported by calculating clustering scores from the free recall protocols. Imagery subjects demonstrated a greater tendency to organize their output according to the input groupings than did the nonimagery subjects $(t=1.9, \quad d f=24)$. Further inspection of the data revealed that imagery subjects were more likely to recall all four items from a group, given that they recalled a single item $(t=2.6, d f=24)$. The data clearly suggest a better integrated representation when imagery was used.

Provision of strong-associate retrieval cues improved recall relative to noncued recall $[\mathrm{F}(1,49)=15.86$, MSe $=12.88]$. This effect is almost entirely attributable to the large effect of cuing the uninstructed subjects, as evidenced by the significant interaction of Cue Condition by Encoding Condition $[F(1,49)=13.50$, $\mathrm{MSe}=12.88]$.

Experiment 2 provides support for the hypothesis that the quality of the initial representation influences subsequent retrieval. A well integrated context seriously restricts the usefulness of alternate retrieval methods. The imagery subjects, who combined groups of words into well integrated images, did quite well on free recall; however, when provided with cues, they were forced to abandon their efficient encoding strategy. The disruption of the encoding context prevented the cues from having the beneficial effects that they had for noninstructed subjects. This is not to say that imagery subjects could not use the cues; in fact, the cues did not really harm their performance. The point is, however, that the cues were not useful. The extra information provided by the cues failed to improve performance. This failure stands in marked contrast to the nearly $75 \%$ increase for the subjects without imagery instructions.

Consistent with the encoding-specificity interpretation, the imagery subjects were unable to improve performance with the nonencoded cues. On the other hand, the clear advantage of cues for subjects without imagery instructions is inconsistent with the notion of encoding specificity unless one makes the unlikely assumption that subjects normally encode with respect to high associates. Thus, when the encoding context is well integrated, the material is well learned with respect to that context, but nonencoded retrieval cues are not particularly useful. However, when subjects are not given sufficient opportunity to form a well integrated representation, they exhibit greater flexibility in their ability to use alternative retrieval cues.

\section{EXPERIMENT 3}

Although the results of Experiment 2 demonstrate that extralist cues are more effective for less integrated contexts, the effect is relative. An absolute decrement such that imagery instructions led to better free recall but worse cued recall was not obtained. The problem seemed to be that good encoding led to both a better retrieval plan and stronger individual-item memories. The differences of individual-item memory prevented the good context subjects from performing worse than the poorer context subjects. In an attempt to demonstrate an absolute cuing effect, Experiment 3 employed a paradigm which manipulated amount of integration among items while holding individual-item strength relatively constant.

In the third experiment, subjects studied and recalled a single list of words for four trials. Within the list, some of the words appeared in fixed positions across trials, while the other words were randomly arranged. On the first three trials, subjects recalled in serial order; thus, they attempted to recall the fixed-order words in the same order on every trial, but they were required to recall randomly ordered words in a different way on each trial. Following the fourth presentation, subjects were not asked to recall serially, but rather were provided with strong associates in a cued recall task. Latencies were recorded between the presentation of each cue and the onset of responding with a list item. It was expected that the extent of integration would influence retrieval times. Words studied in a fixed order over several trials should be more locked into their representations, existing only as part of a tightly integrated string. Words studied in a random order over trials should not be so contextually embedded, and, consequently, they should be easier to retrieve using the extralist cues. Such expectations should hold for either recall or latency measures. However, since subjects instructed in the use of strong-associate cues are capable of nearly perfect recall, the prediction may only be upheld in the response times.

\begin{abstract}
Method
Materials. The stimulus materials consisted of a single set of 16 concrete nouns selected from those used in the preceding experiments. The words were arranged in a random sequence for the first trial and arbitrarily divided into four equal segments of four words each. Each of the segments was then designated as either fixed (F) or random (R). Words in a fixed segment remained in constant position across the four trials of learning. Words in random segments were assigned to positions within either of the random blocks, with the restrictions that no word appeared in the same position from one trial to the next and that no two words appeared in adjacent positions across trials. Thus, there were two basic stimulus sequences (FRFR and RFRF) which were identical on the first trial but differed across trials according to the blocks in which items remained constant (first and third or second and fourth). This procedure partially confounds particular items with serial position, but counterbalances them with respect to treatment condition (fixed or random).
\end{abstract}

Design. The experiment consisted of a 2 (sequence) by 
2 (encoding condition) by 4 (trials) mixed design. Sequence, the one between-subjects factor, was included to determine whether position of the fixed and random blocks had any effect on performance. That is, primacy and recency effects might interact with encoding condition. Encoding condition was a within-subjects factor; subjects received half the words in a fixed context over trials, and the other half of the words in a variable context. The final within-subjects factor was trials; subjects within sequences studied a different arrangement of the list on each of the four trials. On the first three trials, all subjects recalled the words in serial order. On the critical fourth trial, they were given a cued recall task.

Subjects were 14 introductory psychology students who received credit for their participation. They were run individually in $25-\mathrm{min}$ sessions. The data from one subject had to be eliminated on the fourth trial due to equipment failure.

Procedure. Instructions to subjects specified that four study trials would be given on the same list of words, but that some of the words would be in the same position on each trial, while some of the words would be in different orders. They were told to recall the words in the order they appeared on each particular trial and to guess if they were unsure of the correct position. The words were typed in lowercase letters and were presented on a memory drum at a 2-sec rate. After each presentation, subjects recalled the words in serial order in a booklet with spaces numbered 1 through 16 on each page. This aided subjects in placing words in their correct positions, even if they forgot preceding words.

Subjects studied and recalled for three trials. Following the fourth presentation, subjects were given a cued recall task rather than the serial recall task they were led to expect. The cues were presented in a two-field tachistoscope. Reaction time was measured by a digital clock counter which was activated with stimulus presentation and terminated when subjects' oral responses triggered a voice key. Subjects were given careful instructions on the use of the cues, and practice at activating the voice-key mechanism. The cues were then presented one at a time in the tachistoscope for a 2 -sec duration. Subjects orally responded by giving the appropriate target item. If subjects failed to respond within $10 \mathrm{sec}$, the experimenter presented the next cue. The order of cues was randomized for each subject by shuffling the deck of cue cards. The experimenter recorded the subjects' latencies and responses.

\section{Results and Discussion}

Training. Analysis of the training data revealed only a reliable main effect of blocks $[F(3,36)=38.25$, $\mathrm{MSe}=1.52 \mathrm{]}$, which is attributable to the typical serialposition effect. Items in the first and last block were better recalled than those in the middle blocks, independent of encoding condition. There was a tendency for items in fixed context to be better recalled than those in a random context, although the difference was not reliable $(\mathrm{p}=.13)$.

Cued recall. The number of targets recalled in response to the strong associate cues did not differ as a function of encoding condition. In fact, recall level was extremely high: $91 \%$ of the fixed words and $90 \%$ of the random words were recalled.

The latency data were of primary interest and were subjected to a 2 (sequence) by 2 (encoding condition) analysis of variance. The main effect of sequence was not reliable, nor was its interaction with encoding condition, indicating that the sequence factor served its intended counterbalancing function; the particular sequence of fixed and random blocks did not influence performance. The main effect of encoding condition was reliable $[\mathrm{F}(1,10)=8.61, \mathrm{MSe}=185,717]$. Targets in fixed order were accessed $220 \mathrm{msec}$ slower than targets in random order $(1,595 \mathrm{msec}$ vs $1,375 \mathrm{msec})$.

The results support the prediction that, when words are studied in a fixed sequence, it is difficult to break that context and retrieve via new cues. Words studied in a random sequence are not integrated into a broader context over trials; therefore, accessing the individual items requires less time. The experiment has succeeded in demonstrating an absolute effect of cuing, such that the better integrated the representation, the harder it is to retrieve with external cues. The fact that this effect was only obtained with latencies suggests that the effect may also be present in the preceding experiments, but that level of recall is not a sufficiently sensitive measure.

Taken together, the results of the three experiments provide support for differences in cue effectiveness depending on the goodness of the initial context. However, there is a problem of interpretation which could be raised by proponents of encoding specificity. The authors believe that the present results reflect a retrieval effect and demonstrate that context can restrict the means of retrieval. However, it might be argued that the binding effect of context is strictly an encoding phenomenon. Proponents of this argument would hold that subjects are always restricted to cues encoded at the time of study, and that the present data demonstrate that as subjects create a better integrated representation, they are less likely to think of high associates as potential retrieval cues.

This explanation seems unlikely for three reasons. First, subjects seldom if ever report using a strategy of generating associates when they are studying a list of words. Second, the procedures used in the first experiment were specifically designed by Tulving and associates to avoid subjects' encoding with respect to strong cues. List items were paired with weak associates, and set lists were used to induce weak cue encoding. Third, differences were obtained in the third experiment using a straightforward measure of retrieval time. It would, of course, be possible to argue that subjects were slower to retrieve fixed-list words simply because they were less likely to have encoded the strongassociate cues at time of study. A simple control experiment was conducted with 12 new subjects to determine the plausibility of such an explanation.

The experiment consisted of a replication of the third experiment through the presentation of the fourth study list. Instead of a recall test, however, the subjects were again given the studied words and were asked to write down the first four associates which came to mind. The rationale was that if there are differences in the frequency with which subjects think of the strongassociate cues during encoding, then the differences 
should be reflected in the free association data. Thus, words in the less integrated random blocks should lead to more strong-associate cues than words in the fixed integrated condition. However, analysis of the data revealed no differences in the frequency of generating strong-associate cues as any of the four responses per target. Furthermore, the correct cues were generated less than $50 \%$ of the time (34\% for random and $43 \%$ for fixed), indicating that the associations are not bidirectional. The target words are strong associates of the cues, but the cues are not always strong associates of the targets. Thus, it does not appear that subjects were only able to use cues aroused at time of study. Similarly, it does not seem likely that the differences in retrieval time observed in the third experiment are attributable to differential encoding of the extralist cue words. The control experiment suggests that subjects were not thinking of the cues during encoding and that, therefore, the results of the three experiments can be attributed to differences in retrieval flexibility.

\section{GENERAL DISCUSSION}

The present series of experiments has attested to the flexibility subjects exhibit during retrieval. Nonencoded retrieval strategies can be highly effective in improving recall. At the same time, the experiments have shown that the degree of flexibility is influenced by conditions of encoding. When a well integrated encoding context is established, nonencoded retrieval cues are relatively less effective.

The results have broad implications for learning, which are inconsistent with traditional teaching methods. Students are taught to learn material as well as possible: to integrate, categorize, and organize. Yet, the present data suggest that such mnemonic devices and organizational strategies can be counterproductive. Students who learn the material well can reproduce the information verbatim, but might not show creativity of access. Perhaps educators should focus on methods of remembering which will allow students to overcome the rigidity of encoding. Better yet, students should be taught to learn material flexibly and creatively, using a variety of study methods, so that if output conditions require a somewhat different handling of the information, recall is still possible.

A number of interesting parallels exist between the present research and the more traditional area of problem solving. Investigators of memory are currently preoccupied with the importance of context. However, such effects are not new in the problem solving literature. In fact, the present results are quite predictable from very old data. If a person were given a box, some tacks, and a candle, he would have little difficulty in attaching the candle to a wall. On the other hand, if the tacks were in the box, the problem would become difficult. The candlestick problem is but one example that a well integrated representation can restrict creative retrieval (see Birch \& Rabinowitz, 1951 , for other examples). So, the negative effects of context have been known under a variety of names, such as Einstellung, functional fixedness, and encoding specificity. But the parallel between memory research and problem solving research seems to end at this point. Research on creativity and problem solving has focused on how people overcome context, while the memory literature has begun to postulate insurmountable detriments of context.

Contextual influence on memory has led to the principle of encoding specificity and the concept of a separate episodic memory system (e.g., Tulving, 1972). These concepts do not err by pointing to the effect of context, but by concentrating on the totality of the effect. Encoding conditions restrict, but do not incapacitate, retrievai systems. In fact, every instance of creativity or problem solution demonstrates the ability of humans to transcend context.

\section{REFERENCES}

BAKER, L., \& SANTa, J. L. Semantic integration and context. Memory \& Cognition, 1977, 5, 151-154.

Bilodeau, E. A., \& Howell, D. C. Free association norms. (Catalog No. D210.2:F87) Washington, D.C: U.S. Government Printing Office, 1965.

Birch, H. G., \& RaBinowitz, H. S. The negative effect of previous experience of productive thinking. Journal of Experimental Psychology, 1951, 41, 121-125.

Bousfield, W. A. The occurrence of clustering in the recall of randomly arranged associates. Journal of General Psychology, 1953, 49, 229-240.

Bower, G. H., Lesgold, A. M., \& Tieman, D. G. Grouping operations in free recall. Joumal of Verbal Leaming and Verbal Behavior, 1969, 8, 481-493.

Ciccone, D. S., \& Brelsford, J. W. Encoding specificity: The processing of stimulus attributes. Joumal of Experimental Psychology: Human Learning and Memony, $1975,1,60-64$.

Hunt, R. R., \& Elers, H. D. Recognition memory and degree of semantic contextual change. Journal of Experimental Psychology, 1974, 103, 1153-1159.

JACOBY, L. L. Effects of organization on recognition memory. Journal of Experimental Psychology, 1972, 92, 325-331.

Light, L. L., \& Carter-Sobell, L. Effects of changed semantic context on recognition memory. Journal of Verbal Learning and Verbal Behavior, 1970, 9, 1-11.

MandleR, G. Organization and memory. In K. W. Spence and J. T. Spence (Eds.), The psychology of learning and motivation. New York: Academic Press, 1967.

Pompi, K. F., \& Lachman. R. Surrogate processes in the short-term retention of connected discourse. Journal of Experimental Psychology, 1967, 75, 143-150.

Santa, J. L., \& Lamwers, L. L. Encoding specificity: Fact or artifact. Journal of Verbal Learning and Verbal Behavior. $1974,13,412-423$.

Santa, J. L., \& Lamwers, L. L. Where does the confusion lie?: Comments on the Wiseman and Tulving paper. Journal of Verbal Learning and Verbal Behavior, 1976. 15. 53-57.

Thomson, D. M., \& Tulving, E. Associative encoding and retrieval: Weak and strong cues. Journal of Experimental Psychology, 1970, 86, 255-262.

Tulving. E. Episodic and semantic memory. In E. Tulving 
and W. Donaldson (Eds.), Organization of memory. New York: Academic Press, 1972.

Tulving, E., \& Osler, S. Effectiveness of retrieval cues in memory for words. Journal of Experimental Psychology, $1968,77,593-601$.

Tulving, E.. \& Thomson, D. M. Retrieval processes in recognition memory: Effects of associative context. Journal of Experimental Psychology, 1971, 87, 116-124.

Tulving, E., \& Thomson, D. M. Encoding specificity and retrieval processes in episodic memory. Psychological Review, 1973, 80, 352-373.

Winograd, E., Karchmer, M. A., \& Russell, I. S. Role of encoding unitization in cued recognition memory. Journal of Verbal Learning and Verbal Behavior, 1971, 10, 199-206.

\section{NOTE}

1. The rejection region for all tests reported in this paper is $\mathrm{p}<.05$ unless otherwise stated.

(Received for publication October 26, 1976; revision accepted January $19,1977$. ) 